\title{
La evaluación formativa en el aprendizaje-servicio. Una experiencia en actividades físicas en el medio natural
}

\section{Formative assessment in service-learning.}

\section{An experience in physical activities in natural environment}

\author{
María Luisa Santos-Pastor ${ }^{1}$, L. Fernando Martínez-Muñoz ${ }^{2}$ y Laura Cañadas ${ }^{3}$
}

Fecha de recepción: 23/01/2019; Fecha de revisión: 25/02/2019; Fecha de aceptación: 25/03/2019

Cómo citar este artículo:

Santos-Pastor, M.L., Martínez-Muñoz, L.F., \& Cañadas, L. (2019). La evaluación formativa en el aprendizaje servicio. Una experiencia en actividades físicas en el medio natural. Revista de Innovación y Buenas Prácticas Docentes, 8, 110-118.

\section{Autor de Correspondencia: marisa.santos@uam.es}

\section{Resumen:}

En este trabajo se presenta la propuesta de evaluación de un proyecto de Aprendizaje-Servicio desarrollado en el grado de Ciencias de la Actividad Física y el Deporte de la Universidad Autónoma de Madrid, concretamente en la asignatura de Actividades Físico-Deportivas en el Medio Natural ( $2^{\circ}$ curso), como trabajo grupal-opcional para la evaluación continua del alumnado. El portafolio constituye el instrumento empleado para recoger las evidencias del trabajo realizado por el grupo en sus diferentes fases. El principal propósito se ha centrado en ofrecer una evaluación formativa, basada en la retroalimentación y en los procesos que ayuden al estudiante a conseguir los objetivos pretendidos, asegurando el éxito en sus aplicaciones y reforzando tanto la adquisición como el desarrollo de las competencias profesionales, mediante la aplicación de experiencias a una situación de práctica real. La propuesta desarrollada ha sido muy bien valorada por los estudiantes del Grado, así como por el alumnado y responsables de los contextos de intervención (servicio). A pesar de incidir de forma objetiva sobre las competencias docentes y generar un interesante impacto sobre el servicio prestado, es necesario desarrollar procesos de evaluación viables y sostenibles, teniendo en cuenta la alta carga de trabajo.

Palabras clave: Aprendizaje-Servicio. Evaluación Formativa. Portafolio. Actividad Física.

\section{Abstract:}

In this paper we present the assessment proposal of a Service-Learning Project developed in the Degree of Physical Activity and Sport Sciences at Autonomous University of Madrid, specifically at the subject of Physical Activities in the Natural Environment as an optional group work for the continuous assessment of the students. The portfolio constitutes the instrument used to collect the evidences of the work carried out by the group in its different phases. The main purpose has been to offer a formative assessment, based on feedback and processes that help the student achieve the intended objectives, ensuring success in their applications and reinforcing both the acquisition and development of professional skills through the application of experiences to a real practice situation. The experience developed has been highly valued by the students of the Degree and by the students and responsible for the intervention contexts (service). Despite having an objective impact on teaching competences and generating an interesting impact on the service provided, it is necessary to develop viable and sustainable evaluation processes, taking into account the high workload.

Key Words: Service-Learning. Formative Assessment. Portfolio. Physical Activity

\footnotetext{
1 Universidad Autónoma de Madrid (España), marisa.santos@uam.es; CÓDIGO ORCID https://orcid.org/00000002-4985-0810.

2 Universidad Autónoma de Madrid (España)), f.martinez@uam.es; CÓDIGO ORCID. https://orcid.org/00000001-5209-7527

3 Universidad Autónoma de Madrid (España)), laura.cannadas@uam.es; CódIGO ORCID http://orcid.org/0000-0003-4179-9018.
} 


\section{INTRODUCCIÓN}

La formación universitaria requiere del empleo de recursos metodológicos que contribuyan al desarrollo de las competencias profesionales en las que el alumnado desarrollará su labor profesional. Entre el abanico de posibilidades que existen actualmente, el Aprendizaje-Servicio (ApS) se ha convertido en un modelo formativo cada vez más empleado en la formación inicial. Esto es debido a los múltiples beneficios que ha mostrado tener sobre el aprendizaje y el desarrollo competencial del alumnado. El ApS combina el aprendizaje de los contenidos curriculares con el servicio a la comunidad. Es decir, el alumnado universitario desarrolla las competencias profesionales mientras cubre una necesidad relacionada con esas competencias en un contexto social desfavorecido (Gelmon, Holland, \& Spring, 2018). La efectividad del ApS ha quedado avalada por múltiples razones, entre las que destacamos: la oportunidad para aplicar contenidos teóricos del aula a la realidad de los contextos de práctica; la adquisición de conocimientos funcionales en contextos reales; la posibilidad de que el alumnado se enfrente a situaciones complejas; la apertura hacia nuevas vías laborales, desarrollando la autonomía, creatividad y responsabilidad, así como la responsabilidad social (Tejada, 2013).

Las investigaciones en torno a experiencias de ApS han mostrado numerosos beneficios en el aprendizaje del alumnado universitario, tales como: aprendizajes académicos significativos, mejora de la autoestima y el autoconcepto, la autoeficacia, así como un mayor compromiso social-cívico y un mayor compromiso con la justicia social (Chiva-Bartoll, Pallares-Piquer, \& Gil-Gómez, 2018; Corbatón-Martínez, Moliner, Martí-Puig, Gil-Gómez, \& Chiva-Bartoll, 2015).

Para que todo esto sea posible, el desarrollo de los proyectos de ApS deben estar organizados en torno a las competencias que se pretendan desarrollar y, siempre, en relación con la necesidad a cubrir en el contexto de intervención. Asimismo, adquiere gran importancia el proceso de tutela para el seguimiento del trabajo y el proceso de evaluación, que permite valorar los aprendizajes del alumnado involucrado (FerrónZubillaga, \& Guinot-Viciano, 2012; Gil, \& Chiva, 2014). Es muy importante que exista una alineación entre las finalidades formativas, la metodología que se propone, el ApS en este caso, y el sistema de evaluación que se utilice. Por ello, los procesos de evaluación formativa son fundamentales para potenciar tanto el aprendizaje del alumnado, como la calidad del proyecto que se lleva a cabo.

\subsection{La evaluación formativa en los proyectos de Aprendizaje-Servicio}

La evaluación es un elemento clave en el proceso de enseñanza y aprendizaje. Desde hace años, en la formación universitaria se demanda de una evaluación para el aprendizaje, es decir, una evaluación formativa que permita una mejora continua de los procesos de enseñanza y aprendizaje, donde el alumnado es el centro de interés. La utilización de procesos de evaluación formativa en el ámbito universitario con el objetivo de conseguir aprendizajes más profundos y duraderos, ha sido apoyada por diversas investigaciones (Hortigüela, Palacios, \& López-Pastor, 2018; López-Pastor \& Sicilia, 2017).

En los proyectos de ApS, se acentúa su importancia para mejorar el proceso de enseñanza y aprendizaje y, por tanto, contribuir a aumentar la calidad del proyecto. Para conseguir este objetivo, la evaluación no puede verse reducida a la utilización de un examen o a una calificación final, sino que debe abarcar una serie de acciones desde el comienzo del proceso de enseñanza y aprendizaje (Cañadas, Santos-Pastor, \& Castejón, 2018). En cualquier caso, el sistema de evaluación utilizado, debe hacer partícipe al alumnado sobre su proceso de enseñanza-aprendizaje, además de concretar las tareas que permitirán evidenciar el mismo, definir el proceso de tutorización y seguimiento por parte del profesorado, acompañado de un feedback sobre cómo se está desarrollando dicho proceso, y que culmine con una calificación acorde a 
la calidad del trabajo realizado (Batlle, 2015). Concretamente, una evaluación formativa que contribuya a un mayor desarrollo de los aprendizajes del alumnado en los proyectos de ApS, tendrá las características propuestas en la Figura 1, y desarrolladas a continuación (Cañadas, 2018; Wiliam, \& Leahy, 2015; Wiliam, \& Thompson, 2007):

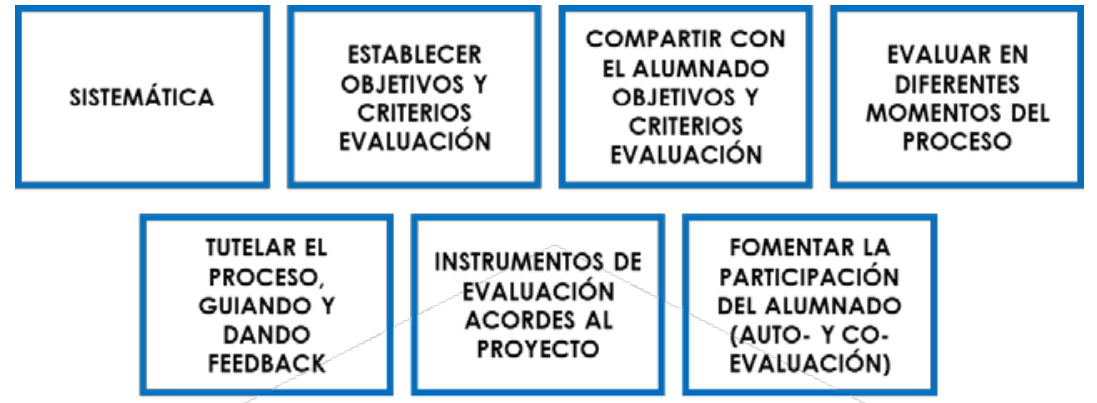

Figura 1. Características de la evaluación formativa en los proyectos de ApS. Fuente: Elaboración propia.

- Sistemática y programada de antemano, de manera que los aprendizajes queden integrados en el proceso de evaluación, y no como algo descontextualizado.

- Establecer claramente los criterios de evaluación del alumnado en el proyecto de ApS. De esta manera quedará fijado lo que se espera que el alumnado aprenda con su participación en el mismo.

- Compartir los objetivos de aprendizaje y los criterios de evaluación con el alumnado, de tal forma que sepa qué van a aprender con su participación en el proyecto y focalicen su atención en los aspectos más importantes.

- Evaluar en diferentes momentos del proceso, de manera que se puedan anticipar adaptaciones y, así, mejorar los aprendizajes que se están desarrollando durante el proyecto, para que al finalizar el mismo se hayan conseguido en el mayor grado posible.

- Tutelar el proceso, guiando y dando feedback al alumnado. Para que los proyectos de ApS se desarrollen asegurando la mayor calidad del servicio y del aprendizaje del alumnado, deben desarrollarse bajo una continua supervisión del profesorado. El alumnado debe recibir información sobre cómo lo está haciendo, en relación con los objetivos establecidos previamente, y qué puede hacer para mejorar el proyecto de ApS que está llevando a la práctica, y, por tanto, sus aprendizajes. De esta manera, podrá introducir cambios antes de que finalice su participación en el mismo.

- Instrumentos de evaluación acordes al proyecto que se va a desarrollar. Los instrumentos de evaluación que se empleen deben permitir que se evalúen todas las competencias que el alumnado debe desarrollar con su participación en el proyecto. Por tanto, en el caso de los proyectos de ApS donde el alumnado va a poner en juego competencias relacionadas con el contenido, pero también con la programación, intervención y evaluación de procesos de enseñanza y aprendizaje, no pueden ser instrumentos estancos y que recojan respuestas memorísticas. Existe una amplia variedad de instrumentos que pueden tener cabida en estos proyectos: portafolios, hojas de observación, fichas de sesiones, trabajos escritos, ensayos, rúbricas, etc.

- Hacer partícipe al alumnado de su evaluación (autoevaluación) y la evaluación de sus compañeros (co-evaluación). La evaluación compartida ayuda al alumnado a ser consciente del proceso desarrollado y a adquirir capacidad para valorar si están consiguiendo los objetivos propuestos, y en qué medida. Estos procesos deben comenzar dando apoyos y ayudas al alumnado para poder llevarlos a cabo, por ejemplo, a través de rúbricas que 
les permitan tener una visión más clara de en qué grado han conseguido un objetivo concreto.

Aplicando estos elementos evaluativos, se espera que los proyectos de ApS contribuyan a que el alumnado adquiera las competencias en un mayor grado, y que esto conlleve un servicio de la mejor calidad posible.

\section{DESARROLLO DE LA EXPERIENCIA DE INNOVACIÓN}

La experiencia que vamos a presentar se enmarca en la metodología de ApS. Concretamente, nos centraremos en la evaluación del alumnado dentro de un proyecto de ApS. El principal propósito fue proponer una evaluación formativa, basada en la retroalimentación y en los procesos que ayudarán al estudiante a conseguir los objetivos pretendidos, asegurando el éxito en sus aplicaciones y reforzando la adquisición y el desarrollo de las competencias profesionales, mediante la aplicación de experiencias a una situación de práctica real.

\subsection{Contexto}

Esta experiencia se desarrolló en la asignatura de Actividades Físico-Deportivas en el Medio Natural (AFDMN) en el $2^{\circ}$ curso del grado en Ciencias de la Actividad Física y el Deporte (CCAFyD) en la Universidad Autónoma de Madrid (UAM). La duración del proyecto, como la de la asignatura, es cuatrimestral. El colectivo con el que se llevó a cabo el ApS fue el alumnado con discapacidad intelectual del Programa Promentor (Programa propio para la inclusión laboral de jóvenes con discapacidad intelectual) de la Fundación Prodis que desarrollaba su labor formativa en la UAM. La experiencia que se presenta en este artículo se llevó a cabo durante el curso 2016/17.

\subsection{Descripción de la experiencia}

Esta experiencia se centra en la propuesta de evaluación del proyecto de ApS en AFDMN como trabajo grupal-opcional para la evaluación continua del alumnado. Esta opción consiste en elaborar un proyecto de ApS relacionado con los contenidos de la materia y llevarlo a la práctica en un contexto en situación de desventaja. El trabajo se desarrolla en grupos de un máximo de 5 estudiantes. El grupo de trabajo se forma antes de la segunda semana de inicio del cuatrimestre. Se distribuyen los roles y las funciones que cada persona asumirá en el trabajo (coordinador/a, secretario/a, otros). Concretamente, en esta experiencia participaron un total de 15 alumnos y alumnas de la titulación de CCAFyD, distribuidos en tres grupos de trabajo. Los receptores del servicio fueron los 30 alumnos y alumnas que cursaban el $1^{\circ}$ y $2^{\circ}$ curso de Promentor.

El proyecto ApS consiste en elaborar un diseño de intervención de actividad físico-deportiva en el medio natural, ponerlo en práctica y evaluarlo. La temática está relacionada con los contenidos específicos de la asignatura y concretan con el profesorado durante las primeras semanas del cuatrimestre. Después de esta primera reunión, se debe entregar a través de la plataforma Moodle un informe sobre la necesidad detectada en relación con la propuesta asignada e ideas previas del grupo para su intervención (punto 2 del portafolio). Tras esta primera fase, y aprobada la viabilidad de la proyecto por parte del tutor, el alumnado desarrollará su propuesta (entre una y tres sesiones prácticas) con el alumnado de Promentor, mientras que el profesorado supervisará y guiará este plan con el objetivo de conseguir un planteamiento de intervención de la mayor calidad posible. Durante la intervención, una de las personas del grupo realiza una recogida de datos mediante una observación estructurada (hoja de observación) y se realizará una grabación, para su posterior análisis. En esta fase, el profesorado asiste presencialmente a cada una de ellas, dando feedback al alumnado de CCAFyD sobre la idoneidad del proceso de intervención y las posibles adaptaciones o cambios necesarios en el desarrollo de la propuesta. Durante todo este proceso, el alumnado irá completando su "diario de aprendizajes", donde 
reflexiona sobre aquellos aspectos más destacados que su participación en el proyecto haya reportado. Por último, tras la intervención, el alumnado realiza una síntesis del trabajo y formula propuestas de mejora en función de la información recogida a lo largo del proceso sobre su proyecto. Realiza un informe individual y grupal sobre lo aprendido, así como su implicación en el mismo.

El portafolio es el instrumento empleado para recoger las evidencias del trabajo realizado por el grupo en sus diferentes fases. La estructurado del mismo y los diferentes apartados quedan reflejados tal y como se recoge en la Figura 2.

\section{- INTRODUCCIÓN}

o Datos del grupo. Roles y funciones.

- Expectativas ante el proyecto. Compromisos del grupo.

- Justificación de los motivos de la selección de esta opción de trabajo.

- DIAGNÓSTICO DE LA NECESIDAD. OBSERVACIÓN INICIAL

o Presentación del grupo Prodis-Promentor. Situación de partida.

o Necesidades detectadas. Valoración inicial en relación al contenido a impartir.

- DESARROLLO PROYECTO

- Evidencias de organización del grupo

- Calendario de trabajo y entregas (reuniones, tutorías, sesiones, etc.)

- Registro de incidencias (cuestiones puntuales o sucesos de interés vinculados con el proyecto)

- Evidencias obligatorias

- Registro y valoración del tiempo dedicado

- Fundamentación teórica: contenido EF, Discapacidad Intelectual. (Revisión de al menos tres referencias bibliográficas y ficha de lectura).

- Ficha de taller y propuesta de sesión

- Hoja de Observación

- Diario de aprendizajes

- Video recopilatorio

- Referencias bibliográficas utilizadas

o Evidencias optativas.

- Material didáctico complementario (fichas, mapas, videos didácticos, etc.)

- Otros. Ampliación de contenidos.

\section{- CONCLUSIONES}

o Síntesis del trabajo realizado.

o Aprendizajes y vivencias.

o Propuesta de mejora.

\section{- EVALUACION}

o Informes de evaluación individual

o Informes de evaluación del grupo

Figura 2. Estructura del portafolio.

Fuente: Elaboración propia.

\subsection{Evaluación de la experiencia}

Para valorar la experiencia sobre el sistema de evaluación formativo empleado para evaluar el aprendizaje del alumnado en el proyecto de ApS, se tuvo en cuenta la percepción del alumnado y del profesorado con respecto a dicho proceso. Se preguntó al alumnado sobre la utilidad de esta experiencia, las ayudas recibidas y la satisfacción con la misma. Estas preguntas, 6 en total, se contestan con una escala Likert de 5 puntos (Nada a Mucho) y se calculó el valor medio de las respuestas (entre 1 y 5).

Por otra parte, se preguntó tanto al alumnado como al profesorado por las características de la experiencia (innovadora, efectiva, sostenible y replicable). En el 
caso del alumnado, a través de un cuestionario y al profesorado a través de respuestas abiertas. La forma de responder era la misma que en las anteriores cuestiones,

También se valoraron las ventajas e inconvenientes de este sistema de evaluación. Al alumnado se le preguntó a través de un cuestionario (32 preguntas) que se contestaba con una escala Likert de 5 puntos (Nada a Mucho). Al profesorado se le solicitó de forma abierta que desarrollase aquellas ventajas e inconvenientes que consideraba que podía tener ese sistema de evaluación.

En todas las respuestas del cuestionario se calculó el valor más repetido (Nada, poco, algo, bastante, mucho).

\section{RESULTADOS}

En este apartado vamos a presentar los resultados de los diferentes aspectos valorados tras la aplicación del sistema de evaluación formativo para evaluar los aprendizajes en el proyecto de ApS.

En la Tabla 1, se recoge la opinión del alumnado sobre la utilidad de la experiencia. Aunque en la respuesta es muy buena en todos los ítems, la media de respuesta para cada ítem está por encima de 3.5. Destaca la valoración que realiza el alumnado sobre la negociación de la experiencia en esa asignatura $(M=4.8)$ y que esa experiencia les haya ayudado a adquirir competencias profesionales ( $M=4.7)$.

Tabla 1.

Opinión del alumnado sobre la utilidad de la experiencia

\begin{tabular}{|c|c|c|c|}
\hline & Bastante* & Mucho & $\begin{array}{c}\text { Media } \\
(1-5)\end{array}$ \\
\hline $\begin{array}{l}\text { ¿Se ha negociado la utilización de esta experiencia en } \\
\text { la asignatura al comienzo del curso }\end{array}$ & & $x$ & 4.8 \\
\hline $\begin{array}{l}\text { ¿Crees que esta experiencia te ha ayudado a adquirir } \\
\text { competencias profesionales? }\end{array}$ & & $x$ & 4.7 \\
\hline $\begin{array}{l}\text { ¿La evaluación que se ha planteado favorece la } \\
\text { adquisición de las competencias profesionales? }\end{array}$ & $x$ & & 3.7 \\
\hline ¿Consideras útil lo aprendido con esta experiencia? & $\mathrm{x}$ & & 3.8 \\
\hline
\end{tabular}

En la Tabla 2, se presenta la valoración media del alumnado sobre las ayudas recibidas y la satisfacción con la experiencia y el sistema de evaluación empleado en la experiencia de ApS. Destaca la satisfacción global con la asignatura $(M=5)$ y la valoración de las ayudas facilitadas por el docente (4.9). De aquí se deduce que el alumnado valora el trabajo y esfuerzo por parte del docente detrás de la experiencia, para llevar un seguimiento y ser una ayuda para el alumnado.

Tabla 2.

Valoración media del alumnado sobre las ayudas recibidas y la satisfacción con la experiencia

\begin{tabular}{lc}
\hline \multicolumn{1}{c}{ Ítems } & $\begin{array}{c}\text { MEDIA } \\
\text { (1-5) }\end{array}$ \\
\hline ¿Cómo valoras las ayudas recibidas por el docente? & 4.9 \\
¿Cómo valoras las ayudas recibidas por los compañeros? & 4.7 \\
Señala la satisfacción global en relación con la experiencia & 5 \\
$\begin{array}{l}\text { Señala la satisfacción global en relación con la evaluación de la } \\
\text { experiencia }\end{array}$ & 4.2 \\
¿Cuál es el grado de dificultad de la experiencia? & 4.6 \\
\hline
\end{tabular}


A continuación, se presentan los resultados sobre las características de la experiencia de evaluación empleada. El alumnado (Tabla 3) valora de forma muy favorable esta experiencia como innovadora, sostenible y replicable (valores por encima de 4.5). Sin embargo, considera que, aun siendo sostenible, podría serlo más ( $M=3.1)$.

Tabla 3.

Valoración del alumnado sobre las características de la experiencia.

\begin{tabular}{lccc}
\hline \multicolumn{1}{c}{ Ítems } & Bastante $^{*}$ & Mucho & Media \\
\cline { 2 - 4 } $\begin{array}{l}\text { Innovadora, porque desarrolla soluciones nuevas o } \\
\text { creativas }\end{array}$ & $\mathrm{X}$ & 4.9 \\
$\begin{array}{l}\text { Efectiva, porque demuestra un impacto positivo y } \\
\text { tangible de mejora }\end{array}$ & $\mathrm{X}$ & 4.8 \\
$\begin{array}{l}\text { Sostenible, porque se mantiene en el tiempo y } \\
\text { puede producir efectos duraderos }\end{array}$ & $\mathrm{X}$ & & 3.1 \\
$\begin{array}{l}\text { Replicable, cuando es posible utilizarla como } \\
\text { modelo para desarrollarla en otros contextos }\end{array}$ & & $\mathrm{X}$ & 4.7 \\
\hline e han eliminado las columnas de Nada, Poco y Algo al no haber respuestas en las mismas.
\end{tabular}

El profesorado, por su parte, considera esta como una experiencia innovadora, ya que se aleja de lo que suele ser común, para dar paso a un aprendizaje experiencial sin ser una práctica simulada o proyecto teórico. Considera también que es una práctica efectiva, porque se ven los resultados y el impacto positivo sobre el grupo con el que se aplica; sostenible, porque genera un impacto en su formación desde la experiencia real y la superación de los obstáculos que van surgiendo; y, replicable, porque podría realizarse en cualquier contexto donde exista una necesidad de intervención.

Por último, vamos a analizar las ventajas e inconvenientes detectados en el sistema de evaluación. En la Figura 4 se recoge la valoración que realiza el alumnado de las posibles ventajas. Encontramos que todos los ítems se valoran como bastante 0 muy de acuerdo por parte del alumnado. Asimismo se puede apreciar la respuesta más repetida por parte del alumnado para cada uno de los ítems estudiados.

\begin{tabular}{lcc}
\hline \multicolumn{1}{c}{ Valoración del alumnado } & Bastante & Mucho \\
\cline { 2 - 3 } 01. Ofrece alternativas a todos los estudiantes & & $\mathrm{X}$ \\
02. Hay un contrato previo, negociado y consensuado del sistema & $\mathrm{X}$ & \\
de evaluación & & $\mathrm{X}$ \\
03. Está centrado en el proceso, importancia del trabajo diario & & $\mathrm{X}$ \\
04. El estudiante realiza un aprendizaje activo & $\mathrm{X}$ & \\
05. Se plantea el trabajo en equipo de forma colaborativa & $\mathrm{X}$ & \\
06. El alumno/a está más motivado, el proceso de aprendizaje es & \\
$\quad$ más motivador & $\mathrm{X}$ & $\mathrm{X}$ \\
07. La calificación es más justa & $\mathrm{X}$ & $\mathrm{X}$ \\
08. Mejora la tutela académica (seguimiento y ayuda al alumno/a) & & $\mathrm{X}$ \\
09. Permite aprendizajes funcionales & & \\
10. Genera aprendizajes significativos & $\mathrm{X}$ & \\
11. Se aprende mucho más & $\mathrm{X}$ & $\mathrm{X}$ \\
12. Mejora la calidad de los trabajos exigidos & & $\mathrm{X}$ \\
13. Hay interrelación entre teoría y práctica & $\mathrm{X}$ \\
14. Evalúa todos los aspectos posibles (en referencia al saber, \\
$\begin{array}{l}\text { saber hacer y saber estar y ser) } \\
\text { 15. Hay retroalimentación en documentos y actividades }\end{array}$ \\
$\begin{array}{l}\text { 16. Hay posibilidad de corregir errores en documentos y actividades } \\
\text { 17. Se da un seguimiento más individualizado }\end{array}$ \\
18. Requiere más responsabilidad & $\mathrm{X}$ & $\mathrm{X}$ \\
\hline
\end{tabular}

Figura 3. Valoración del alumnado de las posibles ventajas del sistema de evaluación.

*Se han eliminado las columnas de Nada, Poco y Algo al no haber respuestas en las mismas. 
El profesorado, por su parte, aporta las siguientes ventajas que considera que tiene esta propuesta:

(1) La propuesta de evaluación desarrollada con los grupos de trabajo de ApS ha supuesto un proceso continuo, lo que ha permitido seguir la evolución de su propuesta e ir ajustándola progresivamente a los propósitos previstos. El acompañamiento, la supervisión y la implicación de la tutela han sido claves para poder engranar una evaluación formativa con repercusiones en el proceso formativo.

(2) Destacan la importancia de este proceso para fomentar el aprendizaje activo de los estudiantes, su participación autónoma y la responsabilidad, tanto al elegir el proceso de aprendizaje que quiere llevar, como el compromiso con el contexto en el que interviene.

(3) La participación es un elemento necesario. La implicación del estudiante genera una motivación intrínseca por aprender.

(4) Se produce un mayor rendimiento sobre todo en lo relativo a calificaciones, habiendo más y mejor correspondencia entre la calificación y el aprendizaje adquirido.

(5) Se aprende más y de diferente manera. Se pierde el aprendizaje técnico y se potencia el didáctico.

(6) Mejora la calidad de los trabajos propuestos, al recibir retroalimentación de los mismos que posibilita hacer mejoras.

(7) Se favorece una interrelación entre la teoría y la práctica mucho más completa.

(8) Las obligatorias retroalimentaciones del trabajo, en sus diferentes fases, generan procesos meta-cognitivos, favoreciendo el auto-aprendizaje, al tener que repensar y reflexionar sobre sus propios conocimientos y tener que volver a replantear sus producciones, si se considera necesario.

En el Figura 4, se recoge la opinión del alumnado sobre los posibles inconvenientes del sistema de evaluación empleado. Encontramos que los 3 ítems en los que el alumnado está más de acuerdo son que exige continuidad, exige mayor esfuerzo y que es una dinámica de trabajo poco conocida y, por tanto, hay falta de hábito.

\begin{tabular}{|c|c|c|c|c|c|}
\hline Ítems & Nada & Poco & Algo & Bastante & Mucho \\
\hline 01. Exige una asistencia obligatoria y activa & & & & $\mathrm{x}$ & \\
\hline $\begin{array}{l}\text { 02. Tiene una dinámica de trabajo poco } \\
\text { conocida, falta de hábito }\end{array}$ & & & & & $\mathrm{x}$ \\
\hline 03. Exige continuidad & & & & & $\mathrm{x}$ \\
\hline 04. Hay que comprenderlo previamente & & & & $\mathrm{x}$ & \\
\hline 05. Exige un mayor esfuerzo & & & & & $\mathrm{x}$ \\
\hline 06. Existe dificultad para trabajar en grupo & & & & $\mathrm{X}$ & \\
\hline 07. Se puede acumular mucho trabajo al final & & $x$ & & & \\
\hline 08. Existe una desproporción trabajo/créditos & & $\mathrm{X}$ & & & \\
\hline $\begin{array}{l}\text { 09. El proceso de calificación es más } \\
\text { complejo y, a veces, poco claro }\end{array}$ & $\mathrm{X}$ & & & & \\
\hline $\begin{array}{l}\text { 10. Genera inseguridad e incertidumbre, } \\
\text { dudas sobre que hay que realizar }\end{array}$ & & & $\mathrm{x}$ & & \\
\hline $\begin{array}{l}\text { 11. Es injusto frente a otros procesos de } \\
\text { evaluación }\end{array}$ & $\mathrm{x}$ & & & & \\
\hline 12. Las correcciones han sido poco claras & $\mathrm{X}$ & & & & \\
\hline 13. La valoración del trabajo es subjetiva & $\mathrm{X}$ & & & & \\
\hline $\begin{array}{l}\text { 14. Exige participar en mi propia evaluación } \\
\text { (autoevaluarse) }\end{array}$ & & & & & $\mathrm{x}$ \\
\hline
\end{tabular}

Figura 4. Inconvenientes que se reconocen en el sistema de evaluación aplicado.

Fuente: Elaboración propia. 
El profesorado, por su parte, considera que aquellos aspectos que generan mayores dificultades son:

(1) La necesidad de hacer tutorías de manera continua genera gran dificultad con la compatibilidad horario de las clases.

(2) Es un proceso continuo que requiere una dedicación permanente, tanto para el estudiante, como para el profesorado que no siempre es posible.

(3) Exige un trabajo continuado y un compromiso muy elevado para el docente y para los estudiantes que compensa cuando descubren los beneficios sobre lo procesos de formación. Esto coincide con lo aportado por el alumnado.

(4) La carga de trabajo es muy elevada, no pudiéndolo hacer factible con muchos grupos de trabajo y un solo docente.

(5) La autonomía para dirigir el proceso de aprendizaje se transfiere a la de evaluarse y calificarse, algo que obliga a marcar pautas para ayudarles a participar en el proceso de evaluación de forma activa, debiendo aprender a evaluarse y, posteriormente, a calificarse

\section{CONCLUSIONES}

La evaluación es un aspecto fundamental en los proyectos de ApS. Con esta propuesta tratamos de mostrar una posibilidad para plantear la evaluación del alumnado en un proyecto específico de ApS. La valoración de esta experiencia es bastante buena, tanto por parte del profesorado, como del alumnado. Se destaca su utilidad para la adquisición de las competencias profesionales y el que sea una experiencia innovadora, efectiva, sostenible y replicable. Destaca que: (i) es un sistema de evaluación que genera aprendizajes funcionales y significativos; (ii) se interrelaciona la teoría y la práctica; (iii) el alumnado recibe retroalimentación y el seguimiento es más individualizado, lo que contribuye a mejorar la calidad de los trabajos exigidos, y; (iv) la calificación es más justa. A pesar de ello, parece que todavía hay que realizar un esfuerzo en hacer comprender al alumnado que, para que existan todos estos beneficios, los procesos de evaluación formativa requieren continuidad y una mayor inversión de tiempo por parte del profesorado y alumnado. Además, parece necesario buscar soluciones que hagan viables y sostenibles estos proyectos, teniendo en cuenta la alta carga de trabajo que suponen, tanto para el alumnado como para el profesorado.

\section{REFERENCIAS}

Batlle, R. (2015). Avaluació dels aprenentatges en els projectes d'aprenentatge servei. Centre Promotor d'Aprenentatge Servei. Recuperado de http://www.aprenentatgeservei.cat/intra/aps/documents/aps_avaluacio_aprenen tatge\%20amb\%20annex.pdf

Cañadas, L. (2018). La evaluación formativa en la adquisición de competencias docentes en la formación inicial del profesorado de Educación Física. Tesis Doctoral Inédita. Madrid: Universidad Autónoma de Madrid.

Cañadas, L., Santos-Pastor, M.L., \& Castejón, F.J. (2018). Evaluación en la formación inicial. ¿Avance o retroceso? Bordón. Revista de Pedagogía, 70(4), 9-21. DOI: 10.13042/Bordon.2018.64434

Chiva-Bartoll, Ó., Pallarés-Piquer. M., \& Gil-Gómez, J. (2018). Aprendizaje-servicio y mejora de la Personalidad Eficaz en futuros docentes de Educación Física. Revista Complutense de Educación, 29(1), 181-197.

Corbatón-Martínez, R., Moliner, L., Martí-Puig, M., Gil-Gómez, J., \& Chiva-Bartoll, Ó. (2015). Efectos académicos, culturales, participativos y de identidad del aprendizaje-servicio en futuros maestros a través de la educación física. Profesorado. Revista de Currículum y Formación del Profesorado, 19(1), 280295. 
Ferrán-Zubillaga, A., \& Guinot-Viciano, C. (2012). Aprendizaje-Servicio: Propuesta metodológica para trabajar competencias. Portularia, XII(EXTRA), 187-195. DOI: 10.5218/PRTS.2012.0020.

Gelmon, S.B., Holland, B.A., \& Spring, A. (2018). Assessing Service-Learning and Civic Engagement: Principles and Techniques. Boston: Campus Compact.

Gil, J., \& Chiva, O. (2014). Una experiencia de aprendizaje-servicio en la asignatura «Bases anatómicas y fisiológicas del movimiento» del Área de Didáctica de la Expresión Corporal. Retos. Nuevas tendencias en Educación Física, Deporte y Recreación, 26, 122-127.

Hortigüela, D., Palacios, A., \& López-Pastor, V.M. (2018). The impact of formative and shared or co-assessment on the acquisition of transversal competences in higher education. Assessment \& Evaluation in Higher Education. https://doi.org/10.1080/02602938.2018.1530341

López-Pastor, V.M., \& Sicilia, A. (2017). Formative and shared assessment in higher education. Lessons learned and challenges for the future. Assessment \& Evaluation in Higher Education, 42(1), https://doi.org/10.1080/02602938.2015.1083535

Tejada, J. (2013). La formación de las compete Wiliam, D., \& Thompson, M. (2007). Integrating assessment with instruction: What will it take to make it work? In C.A. Dwyer (Coord), The Future of Assessment: Shaping Teaching and Learning (pp. 53-82). Mahwah, NJ: Lawrence Erlbaum Associatesncias profesionales a través del aprendizaje-servicio. Cultura y Educación, 25(3), 285-294.

Wiliam, D., \& Leahy, S. (2015). Embedding formative assessment: Practical techniques for K-12 classrooms. West Palm Beach: FL: Learning Sciences International.

Wiliam, D., \& Thompson, M. (2007). Integrating assessment with instruction: What will it take to make it work? In C.A. Dwyer (Coord), The Future of Assessment: Shaping Teaching and Learning (pp. 53-82). Mahwah, NJ: Lawrence Erlbaum Associates. 